\title{
Simulation of Gravity Flow of Granular Materials in Silos*
}

\author{
Pierre A. Gremaud ${ }^{1}$ and John V. Matthews ${ }^{1}$ \\ Department of Mathematics and Center for Research in Scientific Computation, \\ North Carolina State University, Raleigh, NC 27695-8205, USA
}

\begin{abstract}
The problem of determining the steady state flow of granular materials in silos under the action of gravity is considered. In the case of a Mohr-Coulomb material, the stress equations correspond to a system of hyperbolic conservation laws with source terms and nonlinear boundary conditions. A higher order Discontinuous Galerkin method is proposed and implemented for the numerical resolution of those equations. The efficiency of the approach is illustrated by the computation of the stress fields induced in silos with sharp changes of the wall angle.
\end{abstract}

\section{Introduction}

In this paper, the steady state flow of incompressible granular materials under the action of gravity is investigated. Of special interest is the case of flows in silos and bins. Indeed, manufacturing industries routinely store and handle vast quantities of raw materials in granular form. The material is usually retrieved through outlets at the bottom of the containers. Serious difficulties during the withdrawal process are often observed. Those range from dead zones of materials sticking the container's walls to violent vibrations that can cause the complete collapse of the structure. In spite of their commonness, those problems are poorly understood. It is proposed here to analyze numerically the structure and properties of the corresponding flows. This work is, to the authors' knowledge, the first application of a higher order numerical method - a third order Discontinuous Galerkin method- to this type of applications.

The two main physical assumptions, which are discussed in Section 2, are that only established, steady state, flows are considered, and that the material is assumed to be everywhere at yield. Most of the existing work in this field deals with steady state flows in conical hoppers, i.e., when using spherical coordinates, in domains such as

$$
\left\{(r, \theta, \varphi) ; r>0,0 \leq \theta<\theta_{w}, 0 \leq \varphi \leq 2 \pi\right\},
$$

\footnotetext{
* This research was supported by the Army Research Office (ARO) through grants DAAH04-95-1-0419 and DAAH04-96-1-0097, by the National Science Foundation (NSF) through grant DMS-9818900, and by a grant from the North Carolina Supercomputing Center.
} 
which corresponds to an infinite, converging hopper of half opening angle $\theta_{w}$. The attention devoted to this case stems from two reasons. First, in a great number of applications, the containers are indeed axisymmetric, if not downright piecewise conical. Second, as a consequence of the invariance of the domain under the scaling transformation $(r, \theta, \varphi) \mapsto(\lambda r, \theta, \varphi)$ where $\lambda>0$, similarity solutions, the so-called radial solutions, can be constructed. This was first observed by Jenike [6], and has played a fundamental role in the design of industrial hoppers ever since [7], [11]. The radial solutions can be found numerically by solving systems of ordinary differential equations, more precisely, boundary value problems. Their behavior is well documented, see e.g. [4], [8]. One should note, however, that the domain of applicability of such an approach is quite limited [5]. Indeed, the very important case of the junction between conical hoppers of different wall angles for instance, see Figure 1 p.7, clearly cannot be treated under the assumption of radial symmetry. Further, even if the considered domains have the necessary symmetry properties, if and when the radial solutions effectively correspond to approximations of what is observed in practice is not clear. The full system of partial differential equations describing the problems at hand has thus to be solved. This is where our contribution lies.

The model is presented in Section 2. Section 3 is devoted to the description of the numerical method. Computational results are discussed and analyzed in Section 4. Finally, some conclusions and remarks on future work are offered in Section 5.

\section{The model}

The equations governing the time dependent flow of granular material under gravity are derived and analyzed in [12]. Those are found to be linearly illposed in most cases of practical interest. To the authors' knowledge, the situation is not fully understood, mathematically or otherwise. In practice, strongly time-dependent problems are usually observed in conjunction with funnel flows, i.e., flows for which the motion essentially takes place in the central part of the silo. This paper deals exclusively with mass flows, i.e., flows for which all the material is mobilized. In this context, established, steady state flows can be observed.

The spatial domain is assumed to be axisymmetric, but not necessarily right conical. The particles are assumed to have no motion in the axial (or $\varphi)$ direction. Even though this assumption may be counterintuitive to fluid dynamicists, it is an established experimental fact for granular materials. The stress tensor takes the form

$$
T=\left[\begin{array}{ccc}
T_{r r} & T_{r \theta} & 0 \\
T_{r \theta} & T_{\theta \theta} & 0 \\
0 & 0 & T_{\varphi \varphi}
\end{array}\right] .
$$


Neglecting the inertial terms, conservation of momentum yields

$$
\nabla \cdot T=\rho g
$$

in which $\rho$ is the density, taken to be constant, and the vector $g$ is the acceleration due to gravity.

Plastic deformation is assumed everywhere. Constitutive models based on plasticity are conveniently expressed in terms of the principal stresses $\sigma_{i}, i=1,2,3$, i.e., the eigenvalues of the stress tensor $T$. If the principal stresses are ordered $\sigma_{1} \geq \sigma_{2} \geq \sigma_{3}$, then the Mohr-Coulomb yield condition reads

$$
\frac{\sigma_{1}}{\sigma_{3}}=\frac{1+\sin \delta}{1-\sin \delta},
$$

where $\delta$ is the angle of internal friction. This relation can be derived from the law of sliding friction [7], Chap. 3. This condition can be expressed in the original stress variables

$$
\left(T_{r r}-T_{\theta \theta}\right)^{2}+4 T_{r \theta}^{2}=\sin ^{2} \delta\left(T_{r r}+T_{\theta \theta}\right)^{2} .
$$

The Haar-von Karman assumption can be invoked to evaluate the circumferential stress $T_{\varphi \varphi}$. Indeed, the Mohr-Coulomb analysis merely states $\sigma_{1} \geq T_{\varphi \varphi} \geq \sigma_{3}$. For axisymmetric converging hoppers, the Haar-von Karman assumption states that $T_{\varphi \varphi}$ is in fact the major principal stress. One can write the corresponding equations in terms of two unknowns $T_{r r}$ and $T_{r \theta}$

$$
\begin{aligned}
& \partial_{\tau} T_{r r}-\partial_{\theta} T_{r \theta}=f\left(\tau, \theta, T_{r r}, T_{r \theta}\right) \\
& \partial_{\tau} T_{r \theta}-\partial_{\theta} T_{\theta \theta}=g\left(\tau, \theta, T_{r r}, T_{r \theta}\right)
\end{aligned}
$$

where some simplifications result from the use of the new variable $\tau=-\ln r$; we do not bother to rename the stress variables. The right hand side terms are given by

$$
\begin{gathered}
f\left(\tau, \theta, T_{r r}, T_{r \theta}\right)=\frac{3-s}{2} T_{r r}+\cot \theta T_{r \theta}-\frac{3+s}{2} T_{\theta \theta}+\rho g e^{-\tau} \cos \theta \\
g\left(\tau, \theta, T_{r r}, T_{r \theta}\right)=-\frac{1+s}{2} \cot \theta T_{r r}+3 T_{r \theta}+\frac{1-s}{2} \cot \theta T_{\theta \theta}-\rho g e^{-\tau} \sin \theta
\end{gathered}
$$

where we have set $s=\sin \delta$, and, for future reference, $c=\sqrt{1-s^{2}}=\cos \delta$. The equation of state, which relates $T_{\theta \theta}$ to the unknowns $T_{r r}$ and $T_{r \theta}$ is the yield condition (3). It should be noticed that (3) is the equation of a cone in the space $\left(T_{r r}, T_{r \theta}, T_{\theta \theta}\right)$, whose central line bisects the $\left(T_{r r}, T_{\theta \theta}\right)$ plane. The corresponding relation between $T_{\theta \theta}$ and the unknowns is therefore not a proper functional relation, but rather assigns the dependent variables $\left(T_{r r}, T_{r \theta}\right)$ to lie on a manifold: the yield surface. The situation greatly simplifies, however, in the case of converging hoppers which we consider in this paper. Indeed, because the large lateral compression taking place, it can then 
be argued that $T_{\theta \theta}$ lies on the "top" of the yield surface. Accordingly, by solving (3) for $T_{\theta \theta}$, the equation of state completing $(4,5)$ is

$$
T_{\theta \theta}=h\left(T_{r r}, T_{r \theta}\right) \equiv \frac{1+s^{2}}{1-s^{2}} T_{r r}+2 \sqrt{\frac{s^{2}}{\left(1-s^{2}\right)^{2}} T_{r r}^{2}-\frac{1}{1-s^{2}} T_{r \theta}^{2}} .
$$

This corresponds to the so-called passive state [7], [9], as opposed to the active state which is the other solution from (3), obtained by changing the sign in front of the radical in (6); see $\S 5$.

Rewriting (4) as

$$
\partial_{\tau} U+\partial_{\theta} F(U)=G(\tau, \theta, U)
$$

with the obvious notation, one can then analyze the eigenvalues $\lambda_{1,2}$ of the Jacobian $F^{\prime}$. A few calculations lead to

$$
\lambda_{1,2}=\mp \tan \delta \mp \frac{1}{c} \sqrt{\frac{s T_{r r} \mp c T_{r \theta}}{s T_{r r} \pm c T_{r \theta}}} .
$$

A quick analysis [3] reveals the eigenvalues to be real and distinct, provided that one stays "in the cone", i.e., $\left|\frac{T_{r \theta}}{T_{r r}}\right|<\tan \delta$. In other words, the steady state stress equations $(4,5,6)$ form a strictly hyperbolic system of nonlinear conservation laws with source terms. The radial and angular variables $\tau$ and $\theta$ can be thought of as time-like and space-like variables, respectively.

The system has to be completed with "initial" and boundary conditions. The "initial" condition used here corresponds to prescribing the stress high up in the hopper, say, on an " $\tau=$ constant" surface, and solve down from there. Several arguments can be considered to justify the fact that the stress information travels downward, see [3], [7], or [11] for more details. In the calculations presented in this paper, the "initial" condition is computed from the radial stress field; see $\S 4$ for details. Finally, the boundary conditions are given by the law of sliding friction. At any point on the wall, the magnitude of the tangential stress $\left|T_{T}\right|$ is proportional to the magnitude of the normal stress $\left|T_{N}\right|$, i.e.,

$$
\left|T_{T}\right|=\mu\left|T_{N}\right|
$$

where $\mu>0$ is the coefficient of wall friction. In a purely radial geometry, the above boundary condition becomes

$$
T_{r \theta}= \pm \mu T_{\theta \theta} \quad \text { on the walls }
$$

with a "+" sign on one side of the hopper and a "-" sign on the other. Observe that those conditions are nonlinear in the unknowns $T_{r r}, T_{r \theta}$.

At this point, it is worth mentioning that the equations can in fact be somewhat "simplified" by the use of the so-called Sokolovskii variables. Those correspond to the natural parameterization of the conical yield surface. This 
approach was for instance taken in [8] and [9]. However, the use of this nonlinear change of variables has the unfortunate side effect of destroying the conservation form of the equations, losing in this way the ability to compute shocks in any reliable way. This prevents, for instance, the computation of stresses occurring at the junction between conical hoppers of different wall angles, one of the main goals of this work. Further, as is well known, many purely numerical problems also appear when solving systems in nonconservation form.

\section{The algorithm}

For the sake of simplicity, we only describe the algorithm in the case of a conical hopper. The method used is a formally third order Discontinuous Galerkin scheme, see [2] and the references listed therein.

Let $\tau_{0}=-\ln r_{0}$, where $r_{0}$ is the value of the radial variable we start from. Let $\theta_{w}>0$ be the half opening angle, and let $\Delta \theta=\theta_{w} / N$ be the mesh size, $N$ being the number of cells. In this axisymmetric setting, the problem is spatially essentially one-dimensional, and thus no efforts have been made to adapt the mesh. We define

$$
V=\left\{v \in L^{\infty}\left(0, \theta_{w}\right)^{2}:\left.v\right|_{K_{j}} \in P^{k}\left(K_{j}\right)^{2}, j=1, \ldots, N\right\},
$$

where $K_{j}=\left[\theta_{j-1}, \theta_{j}\right]$ is the $j$-th cell, with $\theta_{j}=j \Delta \theta$, and $P^{k}\left(K_{j}\right)$ stands for the space of the polynomials of degree at most $\mathrm{k}$ in $K_{j}$. We use $k=2$ or 3 below; see $\S 4$. A semidiscretization consists of looking for $U_{h}(\tau, \cdot) \in V, \tau>\tau_{0}$, such that $U_{h}\left(\tau_{0}, \cdot\right)=\Pi_{V}\left(U\left(\tau_{0}, \cdot\right)\right)$, where $U\left(\tau_{0}, \cdot\right)$ is an "initial condition", see $\S 4, \Pi_{V}$ is a projection operator into $V$, and

$$
\begin{aligned}
& \frac{d}{d \tau} \int_{K_{j}} U_{h}(\tau, \theta) v(\theta) d \theta+\Delta_{+}\left(v\left(\theta_{j-1 / 2}\right) H_{j-1 / 2}\right) \\
& \quad-\sum_{l=1}^{5} \omega_{l} F\left(U_{h}\left(\tau, \theta_{j, l}\right)\right) \frac{d}{d \theta} v\left(\theta_{j, l}\right) \Delta \theta \\
& \quad=\sum_{l=1}^{5} \omega_{l} G\left(\tau, \theta_{j, l}, U_{h}(\tau, \theta)\right) v\left(\theta_{j, l}\right) \Delta \theta, \quad \forall v \in V, j=1, \ldots, N .
\end{aligned}
$$

In the previous expression, $\Delta_{+}$stands for the usual difference operator, $\Delta_{+} U_{j}=U_{j+1}-U_{j}$ and the coefficients $\omega_{l}$ and the nodes $\theta_{j, l}, l=1, \ldots, 5$, $j=1, \ldots, N$ stem from the use the classical 5-point Gaussian quadrature formula. We use the local Lax-Friedrichs flux

$$
H_{j+1 / 2}=\frac{1}{2}\left(F\left(U_{j+1 / 2}^{-}\right)+F\left(U_{j+1 / 2}^{+}\right)-\alpha_{j+1 / 2}\left(U_{j+1 / 2}^{+}-U_{j+1 / 2}^{-}\right)\right),
$$

where $\alpha_{j+1 / 2}$ is the magnitude of the largest eigenvalue of a properly chosen Roe average matrix $A_{j+1 / 2} \approx\left(\frac{d F}{d U}\right)_{U=U_{j+1 / 2}}$, see [3] for details and [10] for 
background. The mass matrix can be made diagonal by choosing the basis functions as Legendre polynomials over each cell [1].

The coefficients of $U_{h}(\tau, \cdot)$ can then be grouped in a vector $\mathcal{U}(\tau)$. The unknown vector $\mathcal{U}(\tau)$ satisfies the following system of ODEs

$$
\frac{d}{d \tau} \mathcal{U}=\mathcal{F}(\mathcal{U})+\mathcal{G}(\tau, \mathcal{U})
$$

where $\mathcal{F}(\mathcal{U})$ and $\mathcal{G}(\tau, \mathcal{U})$ come respectively from the discretization of $F(U)$ and $G(\tau, \theta, U)$ in (7). Note that we use below an unsplit approach. This is justified first by the fact that the source term $\mathcal{G}(\tau, \mathcal{U})$ is not stiff and second, by the realization that the delicate interplay between $\mathcal{G}(\tau, \mathcal{U})$ and the nonlinear boundary conditions would render the implementation of a split algorithm a lot more involved than the present approach.

The discretization with respect to $\tau$ involves a third order TVD RungeKutta [13] combined with a local slope limiting process. Let $\Delta \tau>0$ be a constant increment in $\tau$ and let $\tau^{n}=\tau_{0}+n \Delta \tau$; the algorithm reads then as follows, see e.g. [2]

- Set $U^{(0)}=\Lambda \Pi\left(U_{h}\left(\tau_{0}, \cdot\right)\right)$;

- For $n=0, \ldots, N-1$, compute $U_{h}^{n+1}$ :

1. set $U^{(0)}=U_{h}^{n}$;

2. for $i=1, \ldots, 3$, compute the intermediate stages

$$
U^{(i)}=\Lambda \Pi\left(\sum_{j=0}^{i-1} \alpha_{i j} U^{(j)}+\Delta \tau \beta_{i j}\left(\mathcal{F}\left(U^{(j)}\right)+\mathcal{G}\left(\tau^{n}+d_{j} \Delta \tau, U^{(j)}\right)\right)\right)
$$

3. set $U_{h}^{n+1}=U^{(3)}$.

The numerical parameters $\left\{\alpha_{i j}\right\},\left\{\beta_{i j}\right\}$ and $\left\{d_{j}\right\}, i=1,2,3, j=0,1,2$ are

\begin{tabular}{|c|c|}
\hline 1 & 1 \\
\hline $3 / 41 / 4$ & $01 / 4$ \\
\hline
\end{tabular}
respectively defined as

A thorough description of the local slope limiting operator $\Lambda \Pi$, which is based on the use of a corrected minmod function, can be found, e.g., in [2]; it is not repeated here. Note that both $\Lambda \Pi$ and the proper implementation of the boundary conditions (9) require transformation to the characteristic fields, see [3] for implementation details pertaining to the present application.

\section{Computational results}

We analyze the influence of abrupt changes in the wall angle on the stress field. The geometrical situation is illustrated in Figure 1. 


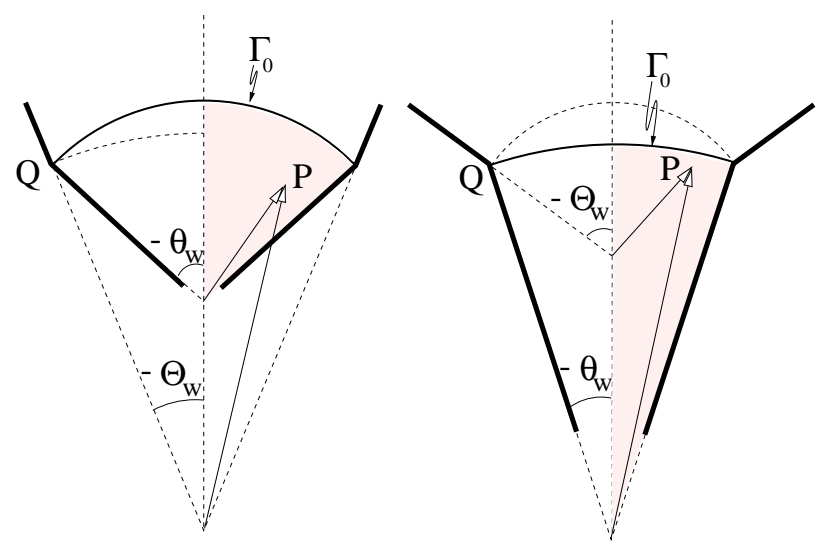

Fig. 1. Geometrical situation; left: transition to a flatter wall angle, right: transition to a steeper wall angle. The radial stress is used to generate an initial condition on the curve $\Gamma_{0}$. The domains of calculation are shaded.

Any point $P$ admits two representations, namely, $(R, \Theta)$ and $(r, \theta)$, corresponding to the natural coordinate systems for the upper and lower hopper respectively. The transition is located through the point $Q$, see again Figure 1, where $Q=\left(R_{0},-\Theta_{w}\right)=\left(r_{0},-\theta_{w}\right)$. For given values of the material parameters $\delta$ and $\mu$, the numerical approach consists then in

- generating the radial stress field $T$ in the upper hopper $\{(R, \Theta): R>$ $\left.0,|\Theta| \leq \Theta_{w}\right\}[4],[7]$; by construction, at a point $(R, \Theta)$, the radial stress field is given by $R T(\Theta)$;

- interpolating the radial stress field on the curve $\Gamma_{0}=\{(r, \theta): r=$ $\left.r_{0},|\theta| \leq \theta_{w}\right\}$, leading to a stress tensor $S_{0}$;

- changing to the new coordinate system through $\mathcal{R}^{T}(\Theta-\theta) S_{0} \mathcal{R}(\Theta-\theta)$, where $\mathcal{R}(\alpha)$ is the rotation matrix of angle $\alpha$;

- solving in the lower hopper $\left\{(r, \theta): 0<r<r_{0}, 0<\theta<\theta_{w}\right\}$ using the algorithm described in $\S 3$.

Notice that by (anti)symmetry, one can solve in one half of the domain only, the law of sliding friction (9) valid on the walls being replaced by the symmetry condition $T_{r \theta}=0$ on the central line.

Some comments are in order. First, the fact that the initial condition is generated from the radial stress field implicitly assumes that this very solution is sought and realized by the problem in the upper part of the hopper. Second, it also takes as granted that the radial solution reaches the curve $\Gamma_{0}$ unperturbed by the wall corner. This last point is clearly satisfied, assuming again a downward propagation of the information for the stresses, in case of a transition to a flatter hopper, i.e., $\Theta_{w}<\theta_{w}$, see Figure 1, left. If the transition is to a steeper hopper, $\theta_{w}<\Theta_{w}$, see Figure 1, right, a quick analysis based 
on the characteristic curves reveals that the difference in angles should not be too large, namely

$$
\Theta_{w}-\theta_{w}<\arctan \left|\frac{1}{\lambda_{\max }}\right|, \quad \theta_{w}, \Theta_{w}>0
$$

where $\lambda_{\max }$ is the largest eigenvalue in modulus of $F^{\prime}$, see (8), evaluated for the radial stress field on the curve $\left\{\left(R_{0}, \Theta\right):|\Theta| \leq \Theta_{w}\right\}$. Third, the case $\theta_{w}=\Theta_{w}$ can be used to check that the algorithm effectively preserves the radial solution. The interaction between the boundary conditions and the forcing terms renders this numerically delicate. Further, the radial solution itself may be unstable [4]. The present approach preserves the radial solution with a great degree of accuracy, see [3] for more details. Finally, it should be noted that the above initial condition is not consistent with the boundary condition (9), unless $\Theta_{w}=\theta_{w}$.

In the calculations below, the opening angles $\Theta_{w}$ and $\theta_{w}$ are respectively taken as $15^{\circ}$ and $10^{\circ}$ in a first experiment, and as $10^{\circ}$ and $12^{\circ}$, in a second. The material parameters correspond to the case of corn in a steel hopper. The angle of internal friction $\delta$ is $32.1^{\circ}$, while the angle of wall friction is $11.7^{\circ}$, in other words, the wall friction is $\mu=\tan 11.7^{\circ}$.

Figure 2 corresponds to the case of a transition to a steeper hopper, whereas in Figure 3, the transition is to a shallower hopper. Some predictions about such transitions can be found in the literature, see e.g. [7], $\S 7.12$. Considerations based on analogy with corresponding Fluid Dynamics problems and/or on the use of radial solutions have been advanced, predicting smooth "rarefaction" waves for transitions to steeper hoppers, and shocks in the case of transitions to wider ones. We note here that neither of those types of arguments can be fully justified. The present results shed some light on this problem.

First, in the case of a transition to a steeper hopper, the results reported in Figure 2 clearly show that there is indeed formation of a rarefaction wave. One can observe, however, that after the waves generated on opposite sides start to interact, they sharpen considerably and shocks appear. In Figure 2 , both the $P^{1}$ and $P^{2}$ cases are reported. Although the $P^{2}$ results offer a slightly better resolution, they also suffer from small oscillations that can be observed around the center line. The small oscillations stem from numerical difficulties linked to the form of the forcing terms there, see the $\cot \theta$ term in (5), and the delicate interplay with the limiting procedure. Such oscillations were not observed for the $P^{1}$ case.

In the case of a transition to a shallower domain, the results of Figure 3 show the immediate formation of shocks in the stress field. In this case, the oscillations of the $P^{2}$ calculations are more pronounced; we only display the $P^{1}$ results. A more in depth analysis of those issues is presented [3]. 

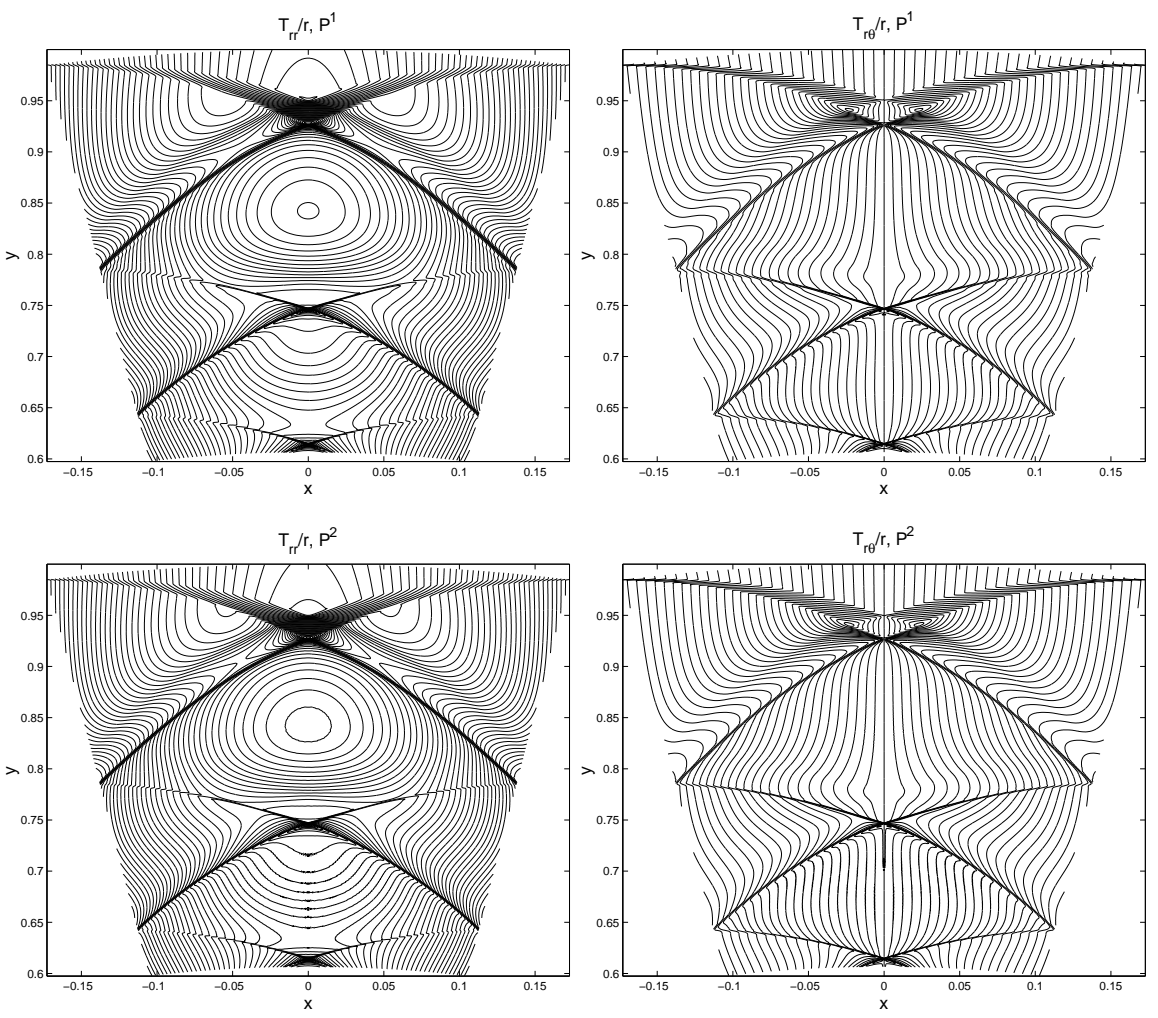

Fig. 2. Structure of the stress field induced by a transition from an opening angle of $15^{\circ}$ to one of $10^{\circ}$. Values of the parameters: $\delta=32.1^{\circ}, \mu=\tan \left(11.7^{\circ}\right)$ (corn, steel wall). First row, $P^{1}$ elements; second row, $P^{2}$ elements.
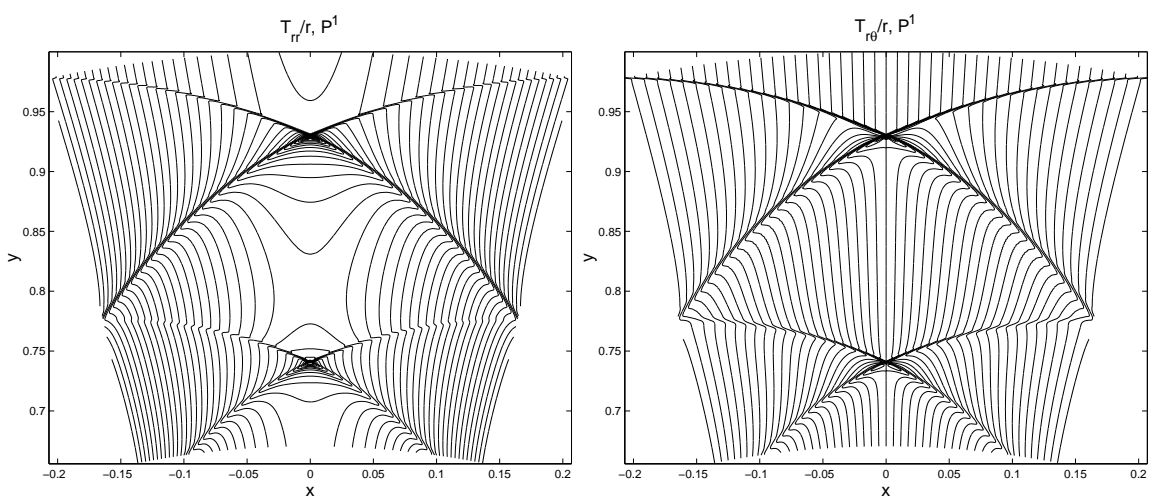

Fig. 3. Structure of the stress field induced by a transition from an opening angle of $10^{\circ}$ to one of $12^{\circ}$. Values of the parameters: $\delta=32.1^{\circ}, \mu=\tan \left(11.7^{\circ}\right)$ (corn, steel wall). $P^{1}$ approximations 


\section{Concluding remarks}

We have presented a numerical study of stress fields induced by the discharge of granular materials in hoppers. The stress equations correspond to a system of hyperbolic conservation laws with several nonstandard features. A higher order Discontinuous Galerkin method has been implemented. The corresponding numerical results partially confirm several "educated guesses" that had been made about the stress field structure induced by changes in the wall angle. A more complete picture of the flow should involve the resolution of the velocity equations, which will be covered in future publications.

\section{Acknowledgments}

The authors would like to thank David Schaeffer and Michael Shearer for many helpful discussions.

\section{References}

1. Cockburn, B., Lin, S.Y., Shu, C.W.: TVB Runge-Kutta local projection discontinuous Galerkin finite element method for conservation laws III: one dimensional systems. J. Comput. Phys. 84 (1989) 90-113

2. Cockburn, B., Shu, C.W.: The Runge-Kutta discontinuous Galerkin method for conservation laws V: multidimensional systems. J. Comput. Phys. 141 (1998) 199-224

3. Gremaud, P.A., Matthews, J.V.: On the computation of hopper flows. In preparation.

4. Gremaud, P.A., Matthews, J.V., Shearer, M.: Similarity solutions for granular flows in hoppers. Proceedings of the SIAM/AMS Conference on Nonlinear PDEs, Dynamics and Continuum Physics, J. Bona, K. Saxton, R. Saxton, Eds., (1998), AMS Contemporary Mathematics Series, to be published.

5. Gremaud, P.A., Schaeffer, D., Shearer, M.: Numerical determination of flow corrective inserts for granular materials in conical hoppers. Submitted to Int. J. Nonlinear Mech.

6. Jenike, A.: Gravity flows of bulk solids. Bulletin No. 108, vol. 52, Utah Eng. Expt. Station, University of Utah, Salt Lake City (1961)

7. Nedderman, R.M.: Static and kinematic of granular materials. Cambridge University Press (1992).

8. Pitman, E.B.: The stability of granular flow in converging hoppers. SIAM J. Appl. Math. 48 (1988) 1033-1052

9. Ravi Prakash, J., Kesava Rao, K.: Steady compressible flow of cohesionless granular materials through a wedge-shaped bunker. J. Fluid Mech. 225 (1991) 21-80

10. Roe, P.L.: Approximate Riemann solvers, parameter vectors, and difference schemes. J. Comp. Phys. 43 (1981) 357-372

11. Royal, A.T.: Private communication. Jenike \& Johanson, Inc. (1998).

12. Schaeffer, D.G.: Instability in the evolution equations describing incompressible granular flow. J. Diff. Eq. 66 (1987) 19-50.

13. Shu, C.W., Osher, S.: Efficient implementation of essentially non-oscillatory shock-capturing schemes. J. Comp. Phys. 77 (1988) 439-471. 